\title{
Synthesis and Characterization of $C d S$ Slim Film Grown by CBD Method
}

\author{
Vhadgal Gorakh Anna \\ PG Student, Department of Physics \\ Padmashri Vikhe Patil College Pravaranagar, Loni
}

\begin{abstract}
Substance shower testimony strategy have been utilized to store Cadmium sulfide dainty film. The affect of the appropriate response temperature and $\mathrm{PH}$ is explored on this work. The introduction of $\mathrm{CdS}$ dainty film Cadmium Sulfate, Anomia, Thiourea and Double refined water is utilized as wellspring of material . Examining Electron microscopy (SEM) is utilized for morphological attributes of CdS slender film. UV spectroscopy have been utilized for optical living arrangements of the CdS slender film .The band hole of CdS dainty film by methods for UV spectroscopy changed into $2.42 \mathrm{eV}$. The $\mathrm{X}-\mathrm{R}$ diffraction investigation is affirmed that the CdS meager film were polycrystline with hexagonal shape the ideal direction of CdS slender film had been(002) and crystalline size 50nM .It changed into chose from the broadenings of corrousponding $\mathrm{X}$-Ray diffraction tops by means of the utilization of Debye scherrer recipe.
\end{abstract}

Keywords:- $C B D, C d S$ thin film, SEM,(scanning electron microscopy), $U V$-spectrocopy, and X-Ray diffractometer.

\section{INTRODUCTION}

Cadmium sulfide (CdS) is one of the most encouraging ll-Vl compound semiconductor material since its wide scope of uses as effective window layer in slight film based sun based cell structure because of its high assimilation coefficient, soundness and low resistivity and various types of hetro intersection sun powered cell, for example, cadmium Telluride, copper indium, gallium diselenide sun based cell and copper indium diselenide.

It has likewise application in different electro-optic gadgets and infra-red gadgets make it appealing semiconductor . Albums slight film can be saved utilizing a few methods, for example, Chemical shower affidavit, synthetic fume testimony, splash pyrolysis, RF faltering and so forth. This procedure Chemical shower affidavit is the exceptionally regular procedure to make slender $\mathrm{CdS}$ slim film favorable position of this strategy is, for example, straightforward, huge territory films, low temperature, and minimal effort technique. CBD technique is the statement of small scale and nanocrystlineCdS have been depicted in writing . Amoung the compound techniques, synthetic shower affidavit is the extraordinary intrigue since it is basic and profoundly productive . CBD permits movable control of size and surface thickness of nanoparticles , and can be utilized for planning of great nanocrystlineCdS films This strategy has been appeared to permit control of mixing period, response time, shower temperature, $\mathrm{pH}$ of arrangement.

Compact discs is a nType semiconducting material with direct band optical band hole $2.42 \mathrm{eV}$ at room temperature .CdS has two kinds of gem structure cubic Zinc blende and hexagonal wurtzite the sorts of is relies upon statement boundary, for example, substrate temperature , $\mathrm{pH}$ convergence of antecedent and so on. Numerous resurches have been contemplating these properties to perceive how the nature of this layer can be improved for better for photovoltaic application .

\section{METHODOLOGY}

The planning of CdS slim film was done by utilizing glass substrate which was at first cleaned by cleanser arrangement and twofold refined water. The CdS flimsy film were saved in arrangement containing .0.1M cadmium sulphate, $0.1 \mathrm{M}$ Thiourea and Anomia arrangement as a complexing operator. $0.1 \mathrm{M} \mathrm{CdSO} 4$ include into the $70 \mathrm{ml}$ refined water and stirr this arrangement continuosly for a few minutes to becomes homogeneous arrangement as a moderate rate fluid smelling salts arrangement was included drop by drop in the answer for keep up the $\mathrm{pH}$ estimation of the arrangement 10 that is arrangement becomes basic.when temp of arrangement arrives at 80 degree Celsius at that point includes the $0.1 \mathrm{M}$ Thiourea after that cleaned glass substrate was submerged in shower during statement process arrangement was no mixed CdS flimsy film was saved for brief at that point test were washing under the ultrasonicator and dry air.

The CdS slight film got were hard, conservative, and great attachment to the glass substrate. 


\section{Reaction}

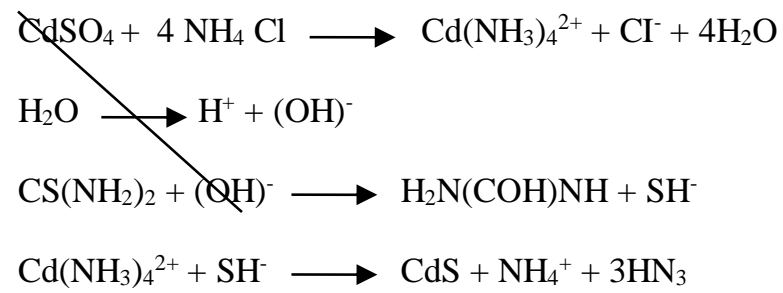

III. SEM STUDIES

Figure shows checking electron microscopy picture of the kept movies demonstrates that CdS meager film is secured totally no splits can be watched .SEM is utilized for the morphological investigation of the CdS slim film. The kept example is circular and moderately uniform surface morphology can be plainly observed . Movies were minimal, uniform, and very well glue to the glass substrate the normal molecule size of CdS dainty film was $0.5 \mathrm{mM}$. The asdeveloped CdS film shading changes with fixation from greenish yellow to splendid yellow. It is seen from SEM pictures of CdS slender film indicated uniform advertisement minimized statement of the substrate.

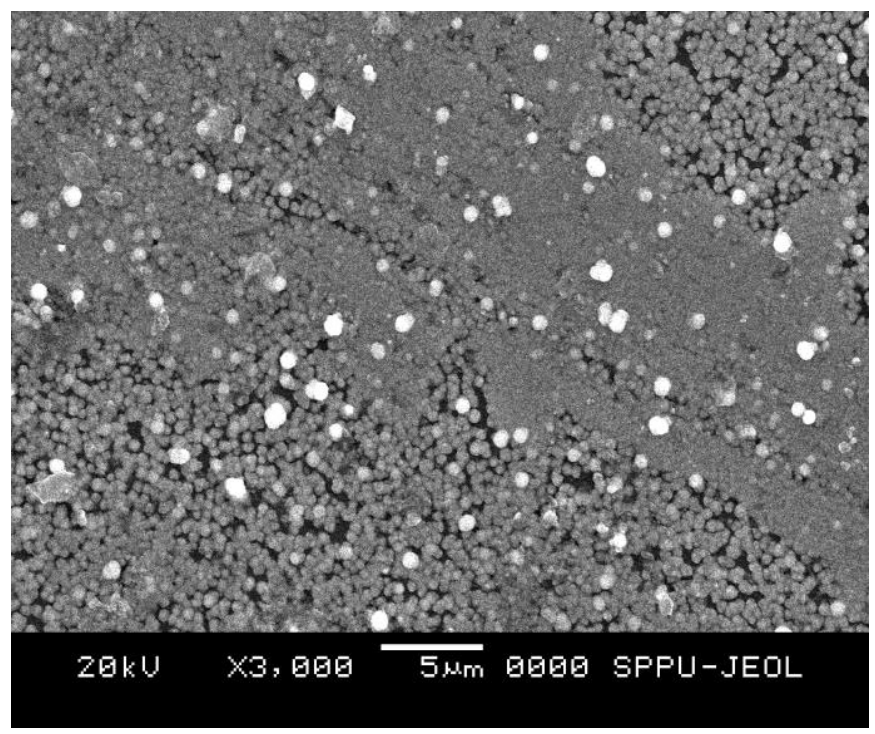

Fig 1:- SEM images for CdS slim film

The greater grain with less limits can be acquired by picking the rough strengthening temperature, surrounding condition and Annealing time. The surface morphology of $\mathrm{CdS}$ dainty movies were considered utilizing the examining electron microscopy (SEM - JOEL JSM 6360 A with a working voltage $20 \mathrm{KV}$ )

\section{UV SPECTROSCOPY STUDIES}

Optical properties of CdS slim movies were considered inthe frequency going from 300 to $800 \mathrm{~nm}$. The optical band hole of the semiconductor is a significant boundary that assumes a significant job in the development of photovoltaic cells. The CdS slight film displays high transmittance and low reflectance in the noticeable locale.

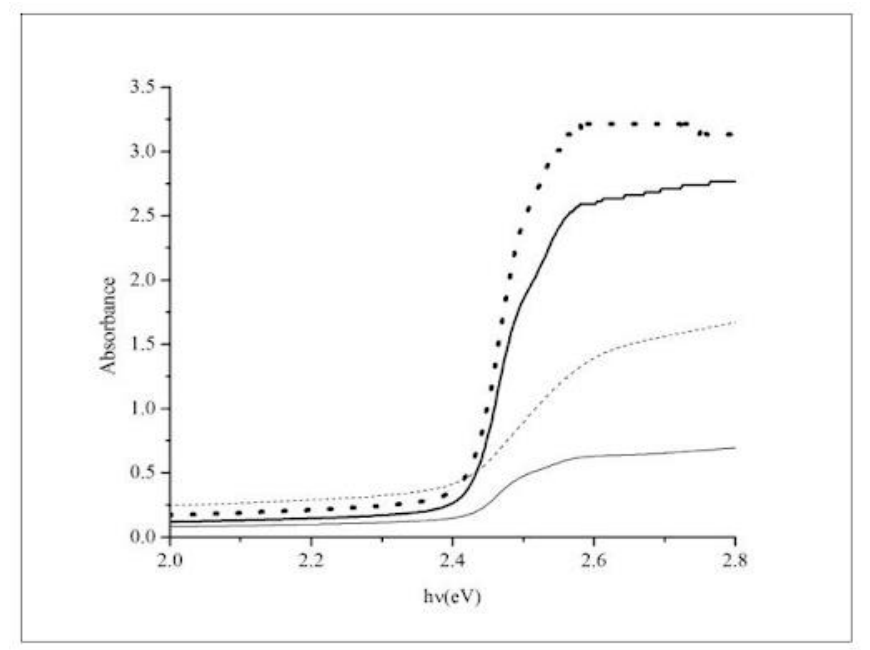

Fig 2:- UV Spectroscopy band gap of CdS slim film

Above figure shows the plots of ahv2 refrains vitality hv for CdS slim movies saved at 80degree celcius the expanded powerful band hole causes nanocrystlineCdS movies to be a progressively reasonable window material in sun powered cell application. The got band hole of CdS dainty film is $2.42 \mathrm{eV}$.

\section{XRD STUDIES}

XRD spectra of the as-stored CdS meager film appeared in figure .XRD investigation for as-kept film has been recorded by X-Ray diffractometer with examining in the 2 theta scope of 20 - 80 degree. The most appealing component of CdS slim film prepered by CBD according to auxiliary perspective is its polymorphism. In XRD investigation uncovers show that a conspicuous wide pinnacle show up at an edge 2theta $=275,28.2,43.7$, and 52.1, which relates to the (100),(002),(101),(110), and (102) planes the outcome is affirmed that the stored CdS slender film were polycrystline in nature with Hexagonal structure having (002) plane as the favored situated. The broadenings of the diffraction tops shows the nanocrystline idea of the examples. The impact of the crystalline size on the electrical and optical conduct of the film is significant. The examples saved at 70 degree have higher pinnacle powers showing better crystallinity the avg crystalline size ( $t$ ) was determined by Debye scherrer recipe 
$\mathrm{t}=0.9$ lambda/BetaCostheta

Where lambda is $\mathrm{X}$ beam frequency (cuka) 1.5409degree angstroms

Theta is the Bragg's diffraction point

The normal crystalline size determined by Debye scherrer equation is $50 \mathrm{~nm}$.

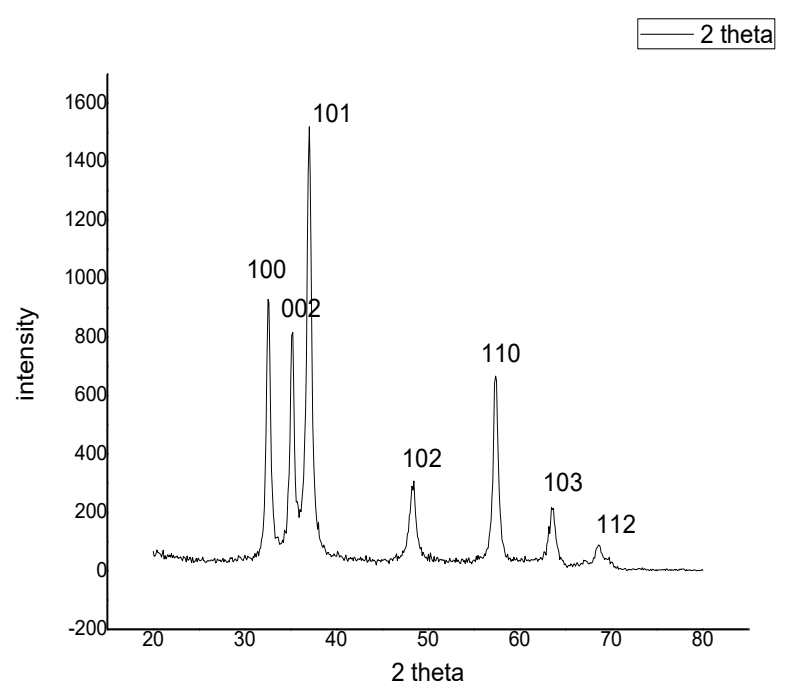

Fig. 3:- XRD Image of CdS slim film

\section{CONCLUSION}

A straightforward and proficient procedures was utilized to CdS slight film utilizing CBD strategy . Cds slim film have been effectively stored on glass substrate utilizing compound shower affidavit strategy. SEM shows the sperical morphology of movies kept dainty film at different temperature. Uniform and conservative film saved in over the whole substrate. Cds dainty movies saved in nearness of smelling salts as complexing operator were near stoichiometry. The film have excellent stoichiometry with about 1 cadmium to sulpher proportion. The band hole was assessed is $2.42 \mathrm{eV}$. The band hole of the film's was seen as expanded after expanding the grouping of Thiourea in the shower. XRD results uncovered that the film's stored at $80^{\circ} \mathrm{c}$ were nanocrystline with hexagonal structure.

\section{REFERENCES}

[1]. Sulbha Kulkarni-Nanotechnology.

[2]. Green MA et.at.al.prog photovoltaic Res.Appl.24, 905913(2016).

[3]. Mayers, , P.V. Structure of a meager film cdTe sun oriented cell 1988,23,59-67.

[4]. Influence of preparing conditions on the optical properties compound kept CdS dainty film A.E.Lgweko.

[5]. Prabha RR, Abdul Khadar M , Bull.Mater.sci31,511(208).

[6]. Lisco F, Kaminski PM, Abbas A, Bass k, Bowers JW , Claudio G, LosurdoM, divider SM, flimsy strong As 582, 323 - 327(2015).

[7]. world cadmium delivering nations world guide site . Accessible online http://www.maps of world .com/minerals/world-cadmiim - makers .html.

[8]. Hani khaalaf an, Isaiah o, Oladeji b, Guangyu chai c, Lee chow , flimsy strong movies 516, 7306-7312(2008).

[9]. Moualkia H, Hariech S, Aida MS, Thin strong Films 518 1259-1262 (2009). 